\title{
Stimulation affecting latex physiology and yield under low frequency tapping of rubber (Hevea brasiliensis) clone RRIM 600 in southern Thailand
}

\author{
Thongchai Sainoi $^{1 *}$, Sayan Sdoodee ${ }^{1}$, Regis Lacote ${ }^{2}$, Eric Gohet ${ }^{3}$, Pisamai Chantuma ${ }^{4}$ \\ ${ }^{1}$ Department of Plant Science, Faculty of Natural Resources, Prince of Songkla University, Hat Yai, 90112, \\ Thailand \\ ${ }^{2}$ CIRAD-UPR Tree Crop-Based Systems, HRPP, Research and Development Building $3^{\text {rd }}$ floor, Kasetsart \\ University, Bangkok 10900, Thailand \\ ${ }^{3}$ CIRAD-UPR Tree Crop-Based Systems, TA-B/34, Montpellier F-34000, France \\ ${ }^{4}$ Chachoengsao Rubber Research Center, RRIT-DOA, Sanam Chai Ket, 24160, Thailand
}

*Corresponding author: noomsainoi@gmail.com

\begin{abstract}
Tapping and ethylene stimulation induces the dynamic change of latex cell metabolism. Those changes under the implement of low frequency tapping systems with ethylene stimulation were investigated during the both periods (low yield and the high yield) of the year of production. The experiment was established at Thepa Research Station, Songkhla province by using 9-year-old rubber trees (clone RRIM 600). An experiment was arranged as One Tree Plot design, there were five treatments following T1: S/3 d1 2d/3, T2: S/2 d2, T3: S/2 d3 ET 2.5\% Pa1(1) 8/y (m), T4: S/3 d2 ET 2.5\% Pa1(1) 4/y (m) and T5: S/3 d3 ET 2.5\% Pa1(1) 12/y (m). There were three replicates in each treatment. It was found that ethylene stimulation affected the responses of initial flow rate (IFR), plugging index (PI), average latex yield (AY) and sucrose content (Suc). There was a significant difference among the treatments in the both periods. After ethylene stimulation, IFR of the T3 and T5 were superior in the low yield period and showed inferior in the high yield period compared with the T1. PI rapidly decreased in the ethylene application treatments in the both periods. AY of the T3 was the highest in the low yield period and AY of the ethylene stimulation treatments was higher than non-stimulated treatments in the high yield period. Suc of the T3 only increased on the first tapping day after stimulation in the both periods. Expression to the ethylene stimulation in the low yield period was higher than the high yield period. It was remarkable that the stimulation was effectively expressed during a full canopy stage. With the positive impact of stimulation on latex physiological parameters, latex yield under low frequency tapping could be compensated by ethylene application.
\end{abstract}

Keywords: Hevea brasiliensis, Initial flow rate, Plugging index, Latex yield, Sucrose content, Stimulation.

Abbreviations: S_spiral cut; d_daily tapping; IFR_initial flow rate; PI_plugging index; AY_average latex yield; Suc_sucrose content; LY_low yield period; HY_high yield period

\section{Introduction}

Rubber tree (Hevea brasiliensis) is an important grobal economic crop yielding natural rubber. It is extensively planted in the Southeast Asia. In Thailand, RRIM 600 clone is grown on $75 \%$ of the rubber plantation in the region (Nakkanong et al., 2008; Pethin et al., 2015), especially in the South of Thailand. The yield is based on latex biosynthesis of rubber trees. Latex is extracted by using a multi-annual tapping system. Linked to the yield per tapping (Lacote et al., 2013), physiological parameters known as Latex Diagnosis or LD (Jacob et al., 1989) are indicators of the potential yield. It is well known that LD parameters can be evaluated under different latex harvesting systems; including tapping frequency and the use of ethylene stimulation (2chloroethylphosphonic acid) (Jacob et al., 1989; d'Auzac et al., 1997; Soumahin et al., 2009; Kudaligama et al., 2010; Lacote et al., 2010; Njukeng et al., 2011; Traore et al., 2011). It has been reported that the yield at each tapping depends on both internal factors (clone and tree age) and external factors (tapping systems, stimulation, the depth, slope and length of tapping cut) (Njukeng et al., 2011; Traore et al., 2011; Gunasekera et al., 2013).

The latex physiological processes involved in latex flow, such as initial flow rate, plugging index, and dry rubber content is closely linked to yield (Gunasekera et al., 2002). Buttery and Boatman $(1964,1966)$ showed that the flow rate is slow down rapidly due to loss in turgor until the flow ceases eventually by the mechanism of latex vessel plugging (Milford et al., 1969). In addition, the diameter and density of latex vessel can assist with obtaining increased yield (Mesquita et al., 2006). Biochemical parameters of latex itself also relate to the yield (Jacob et al., 1989; Gunasekera et al., 2013; Pethin et al., 2015), especially sucrose because latex biosynthesis takes place within the latex vessels using sucrose (Tupy, 1973, 1985; Dusotoit-Coucaud et al., 2009). Latex exudation after tapping causes rapidly initial latexflow. Latex flow duration is normally extended by ethylene stimulation (Yeang, 2005) because of physiological change in the drainage area of the cut after tapping (Gunasekera et al., 2013). The measurement of the latex vessel plugging rate is 
commonly used as the plugging index (Yeang, 2005; Gunasekera et al., 2013). Plugging index is related to many other clonal characters. It is negatively correlated with yield and incidence of dryness and positively correlated with girth, dry rubber content of latex and magnitude of the response to yield stimulation (Waidyanatha and Pathiratne, 1971). It is well known that the low frequency tapping system with stimulation could reduce the risk on rubber production (Soumahin et al., 2009, 2010; Prasanna et al., 2010) and it has been applied to test the efficiency in the South of Thailand. The objective of this investigation was to assess the responses of yield and the changes of biochemical parameters to ethylene stimulation under low frequency tapping comparing with the other tapping intensities.

\section{Results}

\section{The difference of tapping systems on latex physiology}

The changes of initial flow rate (IFR), plugging index (PI), average latex yield (AY) and sucrose content (Suc) according to the different tapping systems and tapping days before and after stimulation in the two periods of study are shown in Table 3. IFR was significantly different each tapping day in both the low yield period and the high yield period of the 5 treatments except on the second tapping day before stimulation in the low yield period. The response of IFR gradually increased during succeeding tapping days. IFR of T3 and T5 with reduced tapping frequency were higher than the other treatments on each tapping day in the low yield period; conversely, these treatments showed lower IFR than that of the other treatments in the high yield period. IFR increased on the first tapping day after stimulation only, of T3 and T5 in the low yield period. Conversely, IFR of ethylene stimulation treatments rapidly increased on the first tapping day after stimulation in the high yield period. Under alternating tapping frequencies, IFR of T2 was higher than $\mathrm{T} 4$ in the both periods.

PI was significantly different on each tapping day of both low yield period and high yield period among the 5 treatments, except the 2 tapping days before stimulation in the low yield period (Table 3). PI responded to tapping and gradually decreased during succeeding tapping days. The highest PI in each tapping day was found for T1 in the low yield period and T2 in the high yield period. Stimulation treatments (T3, T4 and T5) showed lower PI than that of the non-stimulated treatments (T1 and $\mathrm{T} 2$ ) and it rapidly decreased on the first tapping day after stimulation in both the low yield period and the high yield period.

AY showed significant difference each tapping day in the both periods of the 5 treatments except on the second tapping day before ethylene stimulation (Table 3). AY tended to increase during succeeding tapping days. AY of T3, T4 and $\mathrm{T} 5$ were higher than that of $\mathrm{T} 1$ and $\mathrm{T} 2$ for the 3 tapping days after stimulation in the low yield period, with $\mathrm{T} 3$ showed the highest. However, AY of T2 and T4 did not show significant difference on each tapping day. Besides, AY of T3 and T5 immediately increased on the first tapping day after ethylene stimulation. In the high yield period, AY of T4 showed the highest in the 2 tapping days before stimulation and the first tapping day after stimulation, while T3 expressed the highest on the second and the third tapping days after stimulation. In addition, AY in the 3 tapping days after stimulation of $\mathrm{T} 2$, T3, T4 and T5 expressed higher than that of the T1.

Regarding the sucrose content of the latex cells, Suc showed a significant difference in each tapping day in both the low yield period and the high yield periods among the 5 treatments (Table 3). The trend of Suc change was ambiguous. In both periods, Suc of T1 was the highest in the 2 tapping days before stimulation. Suc decreased in all treatments except $\mathrm{T} 3$, on the first tapping day after stimulation. In addition, Suc of T4 and T5 recovered and it was higher than the other treatments on the second and the third tapping days after ethylene stimulation. However, the response to ethylene stimulation of Suc during the high yield period was somewhat less than in the low yielding period.

\section{The variation of season on latex physiology}

Table 4 shows the comparison of IFR and PI between both periods on each tapping day of the 5 treatments. IFR on each tapping day of the T1, T2 and T4 in the low yield period was lower than that of the high yield period, but IFR of the T3 and T5 in the low yield period was higher than the high yield period in the 3 tapping days after stimulation. PI in the 2 tapping days before stimulation in the low yield period was significantly higher than the high yield period of the 5 treatments except the $\mathrm{T} 2$. In the same manner for the 3 tapping days after stimulation, PI of the 5 treatments in the low yield period were significantly higher than that of the high yield period.

Table 5 shows the comparison of AY and Suc between both periods on each tapping day of the 5 treatments. AY on each tapping day in the high yield period was higher than that of the low yield period. On 2 tapping days before stimulation, AY of the 5 treatments in the high yield period was significantly higher than in the low yield period except T5 on the first tapping day before stimulation. On the 3 tapping days after stimulation, AY of T1, T2, T4 and T5 in the high yield period was significantly higher than in the low yield period. But AY of T3 did not show any significant difference in the both periods. Suc of the 5 treatments in the low yield period were higher than that of the high yield period except Suc in $\mathrm{T} 2$.

\section{Percentage changed of latex physiology after stimulation}

Table 6 shows the response of IFR to ethylene stimulation on the 3 tapping days after stimulation of the 5 treatments. In both periods, IFR of T1 did not show significant differences on the 3 tapping days except on the second tapping day in the high yield period. IFR of T2 showed a significant difference on the 3 tapping days. However, the $\mathrm{T} 1$ and $\mathrm{T} 2$ did not receive any stimulation. For the response to ethylene stimulation in both periods, IFR of the T3 showed a significant difference on the 3 tapping days after stimulation except on the second tapping day after stimulation in the high yield period. IFR of the T4 showed no significant difference on the 3 tapping days after stimulation except on the first tapping day after stimulation in the high yield period. IFR of the T5 showed a significant difference on the 3 tapping days after stimulation except on the first tapping day after stimulation in the low yield period.

The response of PI to ethylene stimulation on the 3 tapping days after stimulation of the 5 treatments is illustrated in Table 7. In both periods, PI of T1 did not show a significant difference on the 3 tapping days except on the second tapping day in the low yield period and on the third tapping day in the high yield period. PI of T2 did not show significant differences on the 3 tapping days except on the first and the third tapping days in the high yield period. However, T1 and $\mathrm{T} 2$ did not receive any stimulation. Besides, the response to ethylene stimulation in the both periods showed that PI of T3 was significantly different on the 3 tapping days after 
Table 1. Treatments (tapping system) of the experiment.

\begin{tabular}{|c|c|c|}
\hline Treatments & Tapping system and Description & $\mathrm{TI}^{*}$ \\
\hline \multirow[t]{2}{*}{ T1 } & $\mathrm{S} / 3 \mathrm{~d} 12 \mathrm{~d} / 3$ & 89 \\
\hline & $\begin{array}{l}\text { Third spiral cut downward at daily tapping, two days in tapping followed by one day of tapping } \\
\text { rest in three days }\end{array}$ & \\
\hline \multirow[t]{2}{*}{$\mathrm{T} 2$} & $\mathrm{~S} / 2 \mathrm{~d} 2$ & 100 \\
\hline & Half spiral cut downward at alternate daily tapping & \\
\hline \multirow[t]{2}{*}{ T3 } & $\mathrm{S} / 2 \mathrm{~d} 3 \mathrm{ET} 2.5 \%$ Pa1(1) 8/y (m) & 67 \\
\hline & $\begin{array}{l}\text { Half spiral cut downward at third daily tapping, stimulated with ethephon of } 2.5 \% \text { active ingredient } \\
\text { with } 1 \text { gram of stimulant applied on panel on } 1 \text { centimeter band, } 8 \text { applications per years }\end{array}$ & \\
\hline \multirow[t]{2}{*}{$\mathrm{T} 4$} & $\mathrm{~S} / 3 \mathrm{~d} 2 \mathrm{ET} 2.5 \% \mathrm{~Pa} 1(1) 4 / \mathrm{y}(\mathrm{m})$ & 67 \\
\hline & $\begin{array}{l}\text { Third spiral cut downward at alternate daily tapping, stimulated with ethephon of } 2.5 \% \text { active } \\
\text { ingredient with } 1 \text { gram of stimulant applied on panel on } 1 \text { centimeter band, } 4 \text { applications per years }\end{array}$ & \\
\hline \multirow[t]{2}{*}{ T5 } & $\mathrm{S} / 3 \mathrm{~d} 3 \mathrm{ET} 2.5 \% \mathrm{~Pa} 1(1) 12 / \mathrm{y}(\mathrm{m})$ & 44 \\
\hline & $\begin{array}{l}\text { Third spiral cut downward at third daily tapping, stimulated with ethephon of } 2.5 \% \text { active } \\
\text { ingredient with } 1 \text { gram of stimulant applied on panel on } 1 \text { centimeter band, } 12 \text { applications per } \\
\text { years }\end{array}$ & \\
\hline
\end{tabular}

Table 2. Schedule of tapping and latex sampling.

\begin{tabular}{|c|c|c|c|c|c|}
\hline \multicolumn{2}{|c|}{ Before stimulation ${ }^{[a]}$} & \multirow{2}{*}{$\frac{\text { Stimulation day }^{[\mathrm{c}]}}{\text { Day } 3}$} & \multicolumn{3}{|c|}{ After stimulation $^{[\mathrm{b}]}$} \\
\hline Day 1 & Day 2 & & Day 4 & Day 5 & Day 6 \\
\hline $\begin{array}{l}1^{\text {st }} \text { tapping } \\
\text { Tapping }(-2)\end{array}$ & $\begin{array}{l}2^{\text {nd }} \text { tapping } \\
\text { Tapping }(-1)\end{array}$ & ET & $\begin{array}{c}1^{\text {st }} \text { tapping } \\
\text { Tapping }(+1)\end{array}$ & $\begin{array}{c}2^{\text {nd }} \text { tapping } \\
\text { Tapping }(+2)\end{array}$ & $\begin{array}{c}3^{\text {rd }} \text { tapping } \\
\text { Tapping }(+3)\end{array}$ \\
\hline
\end{tabular}

Table 3. Initial flow rate (IFR) ( $\mathrm{ml} / \mathrm{min})$; plugging index (PI); average latex yield (AY) $(\mathrm{g} / \mathrm{t} / \mathrm{t})$ and sucrose content $(\mathrm{Suc})(\mathrm{mM})$ in each tapping day among the 5 treatments (T1: S/3 d1 2d/3; T2: S/2 d2; T3: S/2 d3 ET 2.5\% Pa1(1) 8/y (m); T4: S/3 d2 ET 2.5\% $\mathrm{Pa} 1$ (1) 4/y (m); T5: S/3 d3 ET 2.5\% Pa1(1) 12/y (m)) in the low yield period and the high yield period.

\begin{tabular}{|c|c|c|c|c|c|c|c|c|c|c|c|}
\hline \multirow[b]{2}{*}{$\mathrm{P}^{[\mathrm{a}]}$} & \multirow[b]{2}{*}{$\mathrm{T}^{[\mathrm{b}]}$} & \multicolumn{5}{|c|}{ Low yield period } & \multicolumn{5}{|c|}{ High yield period } \\
\hline & & $\begin{array}{l}\mathrm{TP}^{[\mathrm{c}]} \\
(-2)\end{array}$ & $\begin{array}{l}\text { TP } \\
(-1) \\
\end{array}$ & $\begin{array}{l}\mathrm{TP} \\
(+1)\end{array}$ & $\begin{array}{l}\text { TP } \\
(+2)\end{array}$ & $\begin{array}{l}\text { TP } \\
(+3)\end{array}$ & $\begin{array}{l}\text { TP } \\
(-2) \\
\end{array}$ & $\begin{array}{l}\text { TP } \\
(-1)\end{array}$ & $\begin{array}{l}\mathrm{TP} \\
(+1)\end{array}$ & $\begin{array}{l}\text { TP } \\
(+2) \\
\end{array}$ & $\begin{array}{l}\text { TP } \\
(+3)\end{array}$ \\
\hline \multirow{5}{*}{ IFR } & T1 & $0.51^{\mathrm{b}}$ & 1.20 & $1.11^{\mathrm{b}}$ & $1.29^{\mathrm{b}}$ & $2.07^{\mathrm{a}}$ & $1.71^{\mathrm{ab}}$ & $1.86^{\mathrm{a}}$ & $1.81^{\mathrm{c}}$ & $2.34^{\mathrm{b}}$ & $2.34^{\mathrm{ab}}$ \\
\hline & $\mathrm{T} 2$ & $0.57^{\mathrm{b}}$ & 0.80 & $1.13^{\mathrm{b}}$ & $1.30^{\mathrm{b}}$ & $1.99^{\mathrm{a}}$ & $1.91^{\mathrm{ab}}$ & $1.91^{\mathrm{a}}$ & $3.00^{\mathrm{a}}$ & $3.67^{\mathrm{a}}$ & $3.11^{\mathrm{a}}$ \\
\hline & $\mathrm{T} 3$ & $0.78^{\mathrm{ab}}$ & 0.88 & $1.78^{\mathrm{a}}$ & $2.05^{\mathrm{a}}$ & $2.39^{\mathrm{a}}$ & $1.34^{\mathrm{b}}$ & $0.97^{\mathrm{b}}$ & $1.51^{\mathrm{d}}$ & $1.51^{\mathrm{c}}$ & $1.56^{\mathrm{b}}$ \\
\hline & $\mathrm{T} 4$ & $0.59^{\mathrm{b}}$ & 0.73 & $0.88^{\mathrm{b}}$ & $0.85^{\mathrm{b}}$ & $1.00^{\mathrm{b}}$ & $2.17^{\mathrm{a}}$ & $1.59^{\mathrm{a}}$ & $2.12^{\mathrm{b}}$ & $1.14^{\mathrm{c}}$ & $1.43^{\mathrm{b}}$ \\
\hline & T5 & $0.92^{\mathrm{a}}$ & 0.82 & $1.27^{\mathrm{ab}}$ & $2.27^{\mathrm{a}}$ & $2.29^{\mathrm{a}}$ & $0.53^{\mathrm{c}}$ & $0.64^{\mathrm{b}}$ & $1.22^{\mathrm{d}}$ & $1.38^{\mathrm{c}}$ & $1.84^{\mathrm{b}}$ \\
\hline \multirow{5}{*}{ PI } & T1 & 7.37 & 7.50 & $6.85^{\mathrm{a}}$ & $4.71^{\mathrm{a}}$ & $5.13^{\mathrm{a}}$ & $5.05^{\mathrm{a}}$ & $2.58^{\mathrm{a}}$ & $2.51^{\mathrm{a}}$ & $2.09^{\mathrm{a}}$ & $1.90^{\mathrm{a}}$ \\
\hline & $\mathrm{T} 2$ & 6.21 & 5.95 & $4.43^{\mathrm{b}}$ & $3.54^{\mathrm{b}}$ & $3.72^{\mathrm{b}}$ & $5.39^{\mathrm{a}}$ & $2.96^{\mathrm{a}}$ & $2.69^{\mathrm{a}}$ & $2.45^{\mathrm{a}}$ & $1.93^{\mathrm{a}}$ \\
\hline & $\mathrm{T} 3$ & 5.46 & 4.98 & $1.64^{\mathrm{c}}$ & $1.35^{\mathrm{c}}$ & $1.45^{\mathrm{c}}$ & $2.18^{\mathrm{b}}$ & $1.57^{\mathrm{b}}$ & $1.18^{\mathrm{b}}$ & $0.87^{\mathrm{b}}$ & $0.90^{\mathrm{b}}$ \\
\hline & $\mathrm{T} 4$ & 7.83 & 6.47 & $2.59^{c}$ & $1.76^{\mathrm{c}}$ & $1.46^{\mathrm{c}}$ & $1.73^{\mathrm{b}}$ & $1.31^{\mathrm{b}}$ & $1.20^{\mathrm{b}}$ & $0.86^{\mathrm{b}}$ & $0.93^{\mathrm{b}}$ \\
\hline & T5 & 5.13 & 6.72 & $2.17^{\mathrm{c}}$ & $2.16^{\mathrm{c}}$ & $2.11^{\mathrm{c}}$ & $2.86^{\mathrm{b}}$ & $1.56^{\mathrm{b}}$ & $1.58^{\mathrm{b}}$ & $0.93^{\mathrm{b}}$ & $1.10^{\mathrm{b}}$ \\
\hline \multirow{5}{*}{ AY } & T1 & $3.39^{b}$ & 7.22 & $8.06^{\mathrm{c}}$ & $13.10^{\mathrm{d}}$ & $18.25^{\mathrm{d}}$ & $16.14^{\mathrm{c}}$ & $36.61^{b}$ & $34.52^{\mathrm{c}}$ & $53.22^{\mathrm{c}}$ & $55.56^{\mathrm{c}}$ \\
\hline & $\mathrm{T} 2$ & $3.86^{\mathrm{b}}$ & 6.43 & $11.81^{\mathrm{c}}$ & $17.47^{\mathrm{cd}}$ & $24.60^{\mathrm{cd}}$ & $18.04^{\mathrm{c}}$ & $35.19^{b}$ & $55.41^{\mathrm{b}}$ & $70.81^{\mathrm{ab}}$ & $69.40^{\mathrm{ab}}$ \\
\hline & $\mathrm{T} 3$ & $6.92^{\mathrm{a}}$ & 8.84 & $50.91^{\mathrm{a}}$ & $68.39^{\mathrm{a}}$ & $71.11^{\mathrm{a}}$ & $26.37^{b}$ & $30.75^{b}$ & $59.09^{\mathrm{ab}}$ & $78.29^{\mathrm{a}}$ & $73.33^{\mathrm{a}}$ \\
\hline & $\mathrm{T} 4$ & $3.98^{\mathrm{b}}$ & 5.50 & $16.97^{\mathrm{bc}}$ & $24.92^{c}$ & $33.94^{c}$ & $47.32^{\mathrm{a}}$ & $56.20^{\mathrm{a}}$ & $66.56^{\mathrm{a}}$ & $57.97^{\mathrm{bc}}$ & $59.78^{\mathrm{bc}}$ \\
\hline & T5 & $7.68^{\mathrm{a}}$ & 5.97 & $28.40^{\mathrm{b}}$ & $45.69^{b}$ & $47.86^{\mathrm{b}}$ & $7.99^{\mathrm{d}}$ & $20.36^{c}$ & $40.17^{\mathrm{c}}$ & $74.18^{\mathrm{a}}$ & $73.04^{\mathrm{a}}$ \\
\hline \multirow{5}{*}{ Suc } & T1 & $9.30^{\mathrm{a}}$ & $7.81^{\mathrm{a}}$ & $3.88^{\mathrm{a}}$ & $3.76^{\mathrm{b}}$ & $2.28^{\mathrm{c}}$ & $5.91^{\mathrm{a}}$ & $3.71^{\mathrm{a}}$ & $1.72^{\mathrm{c}}$ & $2.78^{\mathrm{bc}}$ & $2.52^{\mathrm{bc}}$ \\
\hline & $\mathrm{T} 2$ & $7.01^{\mathrm{ab}}$ & $3.35^{\mathrm{c}}$ & $2.03^{\mathrm{b}}$ & $1.31^{\mathrm{c}}$ & $1.67^{\mathrm{c}}$ & $3.12^{\mathrm{bc}}$ & $3.64^{\mathrm{a}}$ & $2.96^{\mathrm{a}}$ & $2.23^{c}$ & $3.50^{\mathrm{a}}$ \\
\hline & $\mathrm{T} 3$ & - & $3.09^{c}$ & $4.48^{\mathrm{a}}$ & $3.30^{\mathrm{bc}}$ & $3.96^{\mathrm{b}}$ & $1.57^{\mathrm{d}}$ & $1.63^{\mathrm{d}}$ & $1.77^{\mathrm{c}}$ & $2.83^{\mathrm{bc}}$ & $1.89^{\mathrm{c}}$ \\
\hline & $\mathrm{T} 4$ & $5.48^{b}$ & $3.50^{\mathrm{bc}}$ & $1.78^{\mathrm{b}}$ & $6.77^{\mathrm{a}}$ & $7.37^{\mathrm{a}}$ & $2.78^{\mathrm{c}}$ & $2.38^{\mathrm{c}}$ & $1.34^{\mathrm{c}}$ & $4.28^{\mathrm{a}}$ & $2.81^{\mathrm{b}}$ \\
\hline & T5 & $5.05^{\mathrm{b}}$ & $4.51^{\mathrm{b}}$ & $3.53^{\mathrm{a}}$ & $7.91^{\mathrm{a}}$ & $4.93^{\mathrm{b}}$ & $4.02^{\mathrm{b}}$ & $3.01^{\mathrm{b}}$ & $2.34^{\mathrm{b}}$ & $3.09^{\mathrm{b}}$ & $2.72^{\mathrm{b}}$ \\
\hline
\end{tabular}

Note: Means with different letters in the same column indic ate signific ant difference at $P \leq 0.05$ by DMRT. ${ }^{[\mathrm{al}]} \mathrm{P}=\mathrm{Parameters} ;{ }^{[\mathrm{b}]} \mathrm{T}=\mathrm{Treatments} ;{ }^{[\mathrm{c}]} \mathrm{TP}=\mathrm{Tapping} ; \mathrm{TP}(-2,-$ $1)=$ tapping days before ethylene stimulation; $\mathrm{TP}(+1,+2,+3)=$ tapping days after ethylene stimulation. 
Table 4. Initial flow rate $(\mathrm{ml} / \mathrm{min})$ and plugging index in each tapping day between the low yield period (LY) and the high yield period (HY) of the 5 treatments (T1: S/3 d1 2d/3; T2: S/2 d2; T3: S/2 d3 ET 2.5\% Pa1(1) 8/y (m); T4: S/3 d2 ET 2.5\% Pa1(1) 4/y (m); T5: S/3 d3 ET 2.5\% Pa1(1) 12/y (m)).

\begin{tabular}{|c|c|c|c|c|c|c|c|c|c|c|c|}
\hline \multirow[b]{2}{*}{$\mathrm{T}^{[\mathrm{a}]}$} & \multirow[b]{2}{*}{$\mathrm{PR}^{[\mathrm{b}]}$} & \multicolumn{5}{|c|}{ IFR: Initial flow rate (ml/min) } & \multicolumn{5}{|c|}{ PI: Plugging index } \\
\hline & & $\begin{array}{l}\mathrm{TP}^{[\mathrm{c}]} \\
(-2)\end{array}$ & $\begin{array}{l}\mathrm{TP} \\
(-1)\end{array}$ & $\begin{array}{l}\mathrm{TP} \\
(+1)\end{array}$ & $\begin{array}{l}\mathrm{TP} \\
(+2)\end{array}$ & $\begin{array}{l}\mathrm{TP} \\
(+3)\end{array}$ & $\begin{array}{l}\mathrm{TP} \\
(-2)\end{array}$ & $\begin{array}{l}\mathrm{TP} \\
(-1)\end{array}$ & $\begin{array}{l}\mathrm{TP} \\
(+1)\end{array}$ & $\begin{array}{l}\mathrm{TP} \\
(+2)\end{array}$ & $\begin{array}{l}\mathrm{TP} \\
(+3)\end{array}$ \\
\hline \multirow{2}{*}{$\mathrm{T} 1$} & LY & $0.51^{b}$ & 1.20 & $1.11^{b}$ & $1.29^{b}$ & 2.07 & $7.37^{\mathrm{a}}$ & $7.50^{\mathrm{a}}$ & $6.85^{\mathrm{a}}$ & $4.71^{\mathrm{a}}$ & $5.13^{\mathrm{a}}$ \\
\hline & $\mathrm{HY}$ & $1.71^{\mathrm{a}}$ & 1.86 & $1.81^{\mathrm{a}}$ & $2.34^{\mathrm{a}}$ & 2.34 & $5.05^{\mathrm{b}}$ & $2.58^{\mathrm{b}}$ & $2.51^{\mathrm{b}}$ & $2.09^{b}$ & $1.90^{\mathrm{b}}$ \\
\hline \multirow{2}{*}{$\mathrm{T} 2$} & LY & $0.57^{\mathrm{b}}$ & $0.80^{\mathrm{b}}$ & $1.13^{\mathrm{b}}$ & $1.30^{\mathrm{b}}$ & 1.99 & 6.21 & 5.95 & $4.43^{\mathrm{a}}$ & $3.54^{\mathrm{a}}$ & $3.72^{\mathrm{a}}$ \\
\hline & HY & $1.91^{\mathrm{a}}$ & $1.91^{\mathrm{a}}$ & $3.00^{\mathrm{a}}$ & $3.67^{\mathrm{a}}$ & 3.11 & 5.39 & 2.96 & $2.69^{b}$ & $2.45^{\mathrm{b}}$ & $1.93^{\mathrm{b}}$ \\
\hline \multirow{2}{*}{$\mathrm{T} 3$} & LY & 0.78 & 0.88 & 1.78 & 2.05 & $2.39^{\mathrm{a}}$ & $5.46^{\mathrm{a}}$ & $4.98^{\mathrm{a}}$ & $1.64^{\mathrm{a}}$ & $1.35^{\mathrm{a}}$ & $1.45^{\mathrm{a}}$ \\
\hline & HY & 1.34 & 0.97 & 1.51 & 1.51 & $1.56^{\mathrm{b}}$ & $2.18^{\mathrm{b}}$ & $1.57^{\mathrm{b}}$ & $1.18^{\mathrm{b}}$ & $0.87^{\mathrm{b}}$ & $0.90^{b}$ \\
\hline \multirow{2}{*}{$\mathrm{T} 4$} & LY & $0.59^{b}$ & $0.73^{b}$ & $0.87^{b}$ & 0.85 & 1.00 & $7.83^{\mathrm{a}}$ & $6.47^{\mathrm{a}}$ & $2.59^{\mathrm{a}}$ & $1.76^{\mathrm{a}}$ & 1.46 \\
\hline & HY & $2.17^{\mathrm{a}}$ & $1.59^{\mathrm{a}}$ & $2.12^{\mathrm{a}}$ & 1.14 & 1.43 & $1.73^{\mathrm{b}}$ & $1.31^{\mathrm{b}}$ & $1.20^{\mathrm{b}}$ & $0.82^{\mathrm{b}}$ & 0.93 \\
\hline \multirow{2}{*}{ T5 } & LY & 0.92 & $0.82^{\mathrm{a}}$ & 1.27 & $2.27^{\mathrm{a}}$ & 2.29 & $5.13^{\mathrm{a}}$ & $6.72^{\mathrm{a}}$ & $2.17^{\mathrm{a}}$ & $2.16^{\mathrm{a}}$ & $2.11^{\mathrm{a}}$ \\
\hline & HY & 0.53 & $0.64^{b}$ & 1.22 & $1.38^{b}$ & 1.84 & $2.86^{b}$ & $1.56^{\mathrm{b}}$ & $1.58^{b}$ & $0.93^{b}$ & $1.10^{\mathrm{b}}$ \\
\hline
\end{tabular}

Note: Means with different letters in the same column indicate signific ant difference at $P \leq 0.05$ by DMRT. ${ }^{[a]}$ T $=$ Treatments; ${ }^{[b]} \mathrm{PR}=\mathrm{Periods} ;{ }^{[\mathrm{c}]} \mathrm{TP}=\mathrm{Tapping}$; TP $(-2,-1)$ $=$ tapping days before ethylene stimulation; $\mathrm{TP}(+1,+2,+3)=$ tapping days after ethylene stimulation.

Table 5. Average latex yield $(\mathrm{g} / \mathrm{t} / \mathrm{t})$ and sucrose content $(\mathrm{mM})$ in each tapping day between the low yield period (LY) and the high yield period (HY) of the 5 treatments (T1: S/3 d1 2d/3; T2: S/2 d2; T3: S/2 d3 ET 2.5\% Pa1(1) 8/y (m); T4: S/3 d2 ET 2.5\% Pa1(1) 4/y (m); T5: S/3 d3 ET 2.5\% Pa1(1) 12/y (m)).

\begin{tabular}{|c|c|c|c|c|c|c|c|c|c|c|c|}
\hline \multirow[b]{2}{*}{$\mathrm{T}^{[\mathrm{a}]}$} & \multirow[b]{2}{*}{$\mathrm{PR}^{[\mathrm{b}]}$} & \multicolumn{5}{|c|}{ AY: Average latex yield (g/t/t) } & \multicolumn{5}{|c|}{ Suc: Sucrose content (mM) } \\
\hline & & $\begin{array}{l}\mathrm{TP}^{[\mathrm{c}]} \\
(-2)\end{array}$ & $\begin{array}{l}\mathrm{TP} \\
(-1)\end{array}$ & $\begin{array}{l}\mathrm{TP} \\
(+1)\end{array}$ & $\begin{array}{l}\mathrm{TP} \\
(+2)\end{array}$ & $\begin{array}{l}\mathrm{TP} \\
(+3)\end{array}$ & $\begin{array}{l}\mathrm{TP} \\
(-2)\end{array}$ & $\begin{array}{l}\mathrm{TP} \\
(-1)\end{array}$ & $\begin{array}{l}\mathrm{TP} \\
(+1)\end{array}$ & $\begin{array}{l}\mathrm{TP} \\
(+2)\end{array}$ & $\begin{array}{l}\mathrm{TP} \\
(+3)\end{array}$ \\
\hline \multirow{2}{*}{$\mathrm{T} 1$} & LY & $3.39^{\mathrm{b}}$ & $7.22^{\mathrm{b}}$ & $8.06^{\mathrm{b}}$ & $13.10^{\mathrm{b}}$ & $18.25^{b}$ & $9.30^{\mathrm{a}}$ & $7.81^{\mathrm{a}}$ & $3.88^{\mathrm{a}}$ & $3.76^{\mathrm{a}}$ & 2.28 \\
\hline & HY & $16.14^{\mathrm{a}}$ & $36.61^{\mathrm{a}}$ & $34.52^{\mathrm{a}}$ & $53.22^{\mathrm{a}}$ & $55.56^{\mathrm{a}}$ & $5.91^{\mathrm{b}}$ & $3.71^{\mathrm{b}}$ & $1.72^{\mathrm{b}}$ & $2.78^{\mathrm{b}}$ & 2.52 \\
\hline \multirow{2}{*}{$\mathrm{T} 2$} & LY & $3.86^{\mathrm{b}}$ & $6.43^{\mathrm{b}}$ & $11.81^{\mathrm{b}}$ & $17.47^{b}$ & $24.60^{\mathrm{b}}$ & $7.01^{\mathrm{a}}$ & 3.35 & $2.03^{b}$ & $1.31^{b}$ & $1.67^{\mathrm{b}}$ \\
\hline & HY & $18.04^{\mathrm{a}}$ & $35.19^{\mathrm{a}}$ & $55.41^{\mathrm{a}}$ & $70.81^{\mathrm{a}}$ & $69.40^{\mathrm{a}}$ & $3.12^{\mathrm{b}}$ & 3.64 & $2.96^{\mathrm{a}}$ & $2.23^{\mathrm{a}}$ & $3.50^{\mathrm{a}}$ \\
\hline \multirow{2}{*}{$\mathrm{T} 3$} & LY & $6.92^{\mathrm{b}}$ & $8.84^{\mathrm{b}}$ & 50.91 & 68.39 & 71.11 & - & $3.09^{\mathrm{a}}$ & $4.48^{\mathrm{a}}$ & 3.30 & $3.96^{\mathrm{a}}$ \\
\hline & HY & $26.37^{\mathrm{a}}$ & $30.75^{\mathrm{a}}$ & 59.09 & 78.29 & 73.33 & 1.57 & $1.63^{b}$ & $1.77^{\mathrm{b}}$ & 2.83 & $1.89^{\mathrm{b}}$ \\
\hline \multirow{2}{*}{$\mathrm{T} 4$} & LY & $3.98^{\mathrm{b}}$ & $5.50^{\mathrm{b}}$ & $16.97^{b}$ & $24.92^{b}$ & $33.94^{b}$ & $5.48^{\mathrm{a}}$ & 3.50 & 1.78 & $6.77^{\mathrm{a}}$ & $7.37^{\mathrm{a}}$ \\
\hline & HY & $47.32^{\mathrm{a}}$ & $56.20^{\mathrm{a}}$ & $66.56^{\mathrm{a}}$ & $57.97^{\mathrm{a}}$ & $59.78^{\mathrm{a}}$ & $2.78^{\mathrm{b}}$ & 2.38 & 1.34 & $4.28^{\mathrm{b}}$ & $2.81^{\mathrm{b}}$ \\
\hline \multirow{2}{*}{ T5 } & LY & 7.68 & $5.97^{\mathrm{b}}$ & 28.40 & $45.69^{b}$ & $47.86^{b}$ & 5.05 & $4.51^{\mathrm{a}}$ & $3.53^{\mathrm{a}}$ & $7.91^{\mathrm{a}}$ & $4.93^{\mathrm{a}}$ \\
\hline & HY & 7.99 & $20.36^{\mathrm{a}}$ & 40.17 & $74.18^{\mathrm{a}}$ & $73.04^{\mathrm{a}}$ & 4.02 & $3.01^{\mathrm{b}}$ & $2.34^{\mathrm{b}}$ & $3.09^{\mathrm{b}}$ & $2.72^{\mathrm{b}}$ \\
\hline
\end{tabular}

Note: Means with different letters in the same column indicate signific ant difference at $P \leq 0.05$ by DMRT. ${ }^{[a]} \mathrm{T}=$ Treatments; ${ }^{[\mathrm{b}]} \mathrm{PR}=\mathrm{Periods} ;{ }^{[\mathrm{c}]} \mathrm{TP}=\mathrm{Tapping}$; TP $(-2,-1)$ $=$ tapping days before ethylene stimulation; TP $(+1,+2,+3)=$ tapping days after ethylene stimulation.

Table 6. Response of IFR to ethylene stimulation in percentage of the difference in delta $(\% \% \Delta)$ before and after ethylene application in the low yield period and the high yield period among the 5 treatments (T1: S/3 d1 2d/3; T2: S/2 d2; T3: S/2 d3 ET 2.5\% Pa1(1) 8/y (m); T4: S/3 d2 ET 2.5\% Pa1(1) 4/y (m); T5: S/3 d3 ET 2.5\% Pa1(1) 12/y (m)).

\begin{tabular}{|c|c|c|c|c|c|c|}
\hline \multirow{3}{*}{ Treatments } & \multicolumn{6}{|c|}{ IFR: Initial flow rate $(\mathrm{ml} / \mathrm{min})$} \\
\hline & \multicolumn{3}{|c|}{ Low yield period } & \multicolumn{3}{|c|}{ High yield period } \\
\hline & $\% \Delta \mathrm{TP}^{[\mathrm{a}]}(+1)$ & $\% \Delta \mathrm{TP}(+2)$ & $\% \Delta \mathrm{TP}(+3)$ & $\% \Delta \mathrm{TP}(+1)$ & $\% \Delta \mathrm{TP}(+2)$ & $\% \Delta \mathrm{TP}(+3)$ \\
\hline $\mathrm{T} 1$ & $-8.0^{\mathrm{ns}}$ & $7.7^{\mathrm{ns}}$ & $72.0^{\mathrm{ns}}$ & $-2.6^{\mathrm{ns}}$ & $25.9^{* *}$ & $25.8^{\mathrm{ns}}$ \\
\hline $\mathrm{T} 2$ & $42.0^{* *}$ & $62.6^{* *}$ & $150.0^{*}$ & $56.9^{* *}$ & $92.0^{* *}$ & $62.5^{*}$ \\
\hline $\mathrm{T} 3$ & $103.1^{*}$ & $133.3^{*}$ & $173.0^{* *}$ & $56.0^{*}$ & $55.7^{\mathrm{ns}}$ & $61.4^{*}$ \\
\hline T4 & $20.3^{\mathrm{ns}}$ & $17.1^{\mathrm{ns}}$ & $37.0^{\mathrm{ns}}$ & $33.8^{*}$ & $-28.0^{\mathrm{ns}}$ & $-9.8^{\mathrm{ns}}$ \\
\hline T5 & $54.8^{\mathrm{ns}}$ & $176.9^{* *}$ & $179.5^{* *}$ & $91.1^{* *}$ & $116.0^{*}$ & $187.8^{*}$ \\
\hline
\end{tabular}

Note: ${ }^{[a]} \mathrm{TP}=$ Tapping; Data of tapping day after stimulation (TP $(+1)$, TP $(+2)$ and TP (+3)) were compared with tapping day before stimulation $(\mathrm{TP}(-1))$ of the 5 treatments. ns $=$ not significant, $*=$ signific ant $(P \leq 0.05), * *=$ highly signific ant $(P \leq 0.01)$.

Table 7. Response of PI to ethylene stimulation in percentage of the difference in delta $(\% \Delta)$ before and after ethylene application in the low yield period and the high yield period among the 5 treatments (T1: S/3 d1 2d/3; T2: S/2 d2; T3: S/2 d3 ET 2.5\% Pa1(1) 8/y (m); T4: S/3 d2 ET 2.5\% Pa1(1) 4/y (m); T5: S/3 d3 ET 2.5\% Pa1(1) 12/y (m).

\begin{tabular}{|c|c|c|c|c|c|c|}
\hline \multirow{3}{*}{ Treatments } & \multicolumn{6}{|c|}{ PI: Plugging index } \\
\hline & \multicolumn{3}{|c|}{ Low yield period } & \multicolumn{3}{|c|}{ High yield period } \\
\hline & $\% \Delta \mathrm{TP}^{[\mathrm{a}]}(+1)$ & $\% \Delta \mathrm{TP}(+2)$ & $\% \Delta \mathrm{TP}(+3)$ & $\% \Delta \mathrm{TP}(+1)$ & $\% \Delta \mathrm{TP}(+2)$ & $\% \Delta \mathrm{TP}(+3)$ \\
\hline T1 & $-8.6^{\mathrm{ns}}$ & $-37.2^{*}$ & $-31.6^{\mathrm{ns}}$ & $-2.9^{\mathrm{ns}}$ & $-19.2^{\mathrm{ns}}$ & $-26.6^{*}$ \\
\hline $\mathrm{T} 2$ & $-25.6^{\mathrm{ns}}$ & $-40.5^{\mathrm{ns}}$ & $-37.5^{\mathrm{ns}}$ & $-8.8^{* * *}$ & $-17.2^{\mathrm{ns}}$ & $-34.8^{* *}$ \\
\hline $\mathrm{T} 3$ & $-67.0^{* *}$ & $-72.9^{* *}$ & $-70.9^{* *}$ & $-24.8^{*}$ & $-44.7^{* *}$ & $-42.4^{* *}$ \\
\hline $\mathrm{T} 4$ & $-60.0^{* *}$ & -72.7 ** & $-77.5^{* *}$ & $-8.2^{\mathrm{ns}}$ & $-37.8^{*}$ & $-28.7^{\mathrm{ns}}$ \\
\hline T5 & $-67.7^{*}$ & $-68.0^{*}$ & $-68.6^{*}$ & $-1.2^{\mathrm{ns}}$ & $-40.5^{*}$ & $-29.9^{\mathrm{ns}}$ \\
\hline
\end{tabular}

Note: ${ }^{[a]} \mathrm{TP}=$ Tapping; Data of tapping day after stimulation (TP $(+1)$, TP $(+2)$ and TP $\left.(+3)\right)$ were compared with tapping day before stimulation $(\mathrm{TP}(-1))$ of 5 treatments.ns $=$ not significant, $*=$ signific ant $(P \leq 0.05), * *=$ highly significant $(P \leq 0.01)$. 
Table 8. Response of AY to ethylene stimulation in percentage of the difference in delta $(\% \Delta)$ before and after ethylene application in the low yield period and the high yield period among the 5 treatments (T1: S/3 d1 2d/3; T2: S/2 d2; T3: S/2 d3 ET 2.5\% Pa1(1) 8/y (m); T4: S/3 d2 ET 2.5\% Pa1(1) 4/y (m); T5: S/3 d3 ET 2.5\% Pa1(1) 12/y (m).

\begin{tabular}{|c|c|c|c|c|c|c|}
\hline \multirow{3}{*}{ Treatments } & \multicolumn{6}{|c|}{ AY: Average latex yield $(\mathrm{g} / \mathrm{t} / \mathrm{t})$} \\
\hline & \multicolumn{3}{|c|}{ Low yield period } & \multicolumn{3}{|c|}{ High yield period } \\
\hline & $\% \Delta \mathrm{TP}^{[\mathrm{a}]}(+1)$ & $\% \Delta \mathrm{TP}(+2)$ & $\% \Delta \mathrm{TP}(+3)$ & $\% \Delta \mathrm{TP}(+1)$ & $\% \Delta \mathrm{TP}(+2)$ & $\% \Delta \mathrm{TP}(+3)$ \\
\hline T1 & $11.7^{\mathrm{ns}}$ & $81.5^{\mathrm{ns}}$ & $152.9^{* *}$ & $-5.7^{\mathrm{ns}}$ & $45.3^{\text {ns }}$ & $51.7^{*}$ \\
\hline $\mathrm{T} 2$ & $83.6^{*}$ & $171.6^{* *}$ & $282.5^{* *}$ & $57.5^{* *}$ & $101.2^{* *}$ & $97.2^{* *}$ \\
\hline $\mathrm{T} 3$ & $476.0^{* * *}$ & $673.8^{* * *}$ & $704.5^{* * 3}$ & $92.1^{*}$ & $154.6^{* * *}$ & $138.4^{* *}$ \\
\hline $\mathrm{T} 4$ & $208.5^{* * *}$ & $353.1^{* * *}$ & $517.0^{* * *}$ & $18.4^{* * *}$ & $3.2^{\mathrm{ns}}$ & $6.4^{\mathrm{ns}}$ \\
\hline T5 & $375.7^{*}$ & $665.3^{* *}$ & $701.7^{* *}$ & $97.4^{* *}$ & $264.4^{* *}$ & $258.8^{* * *}$ \\
\hline
\end{tabular}

Table 9. Response of Suc to ethylene stimulation in percentage of the difference in delta $(\% \% \Delta)$ before and after ethylene application in the low yield period and the high yield period among the 5 treatments (T1: $\mathrm{S} / 3 \mathrm{~d} 12 \mathrm{~d} / 3$; T2: S/2 d2; T3: S/2 d3 ET 2.5\% Pa1(1) 8/y (m); T4: S/3 d2 ET 2.5\% Pa1(1) 4/y (m); T5: S/3 d3 ET 2.5\% Pa1(1) 12/y (m)).

\begin{tabular}{|c|c|c|c|c|c|c|}
\hline \multirow{3}{*}{ Treatments } & \multicolumn{6}{|c|}{ Suc: Sucrose content (mM) } \\
\hline & \multicolumn{3}{|c|}{ Low yield period } & \multicolumn{3}{|c|}{ High yield period } \\
\hline & $\% \Delta \mathrm{TP}^{[\mathrm{a}]}(+1)$ & $\% \Delta \mathrm{TP}(+2)$ & $\% \Delta \mathrm{TP}(+3)$ & $\% \Delta$ TP $(+1)$ & $\% \Delta \mathrm{TP}(+2)$ & $\% \Delta \mathrm{TP}(+3)$ \\
\hline T1 & $-50.4^{* *}$ & $-51.9^{* *}$ & $-70.8^{* *}$ & $-53.5^{* *}$ & $-25.0^{*}$ & $-32.1^{* * *}$ \\
\hline $\mathrm{T} 2$ & $-39.6^{* *}$ & $-60.9^{* *}$ & $-50.3^{* *}$ & $-18.8^{*}$ & $-38.7^{* *}$ & $-3.8^{\mathrm{ns}}$ \\
\hline T3 & $45.2^{* *}$ & $7.0^{\mathrm{ns}}$ & $28.2^{* *}$ & $8.5^{\mathrm{ns}}$ & $73.5^{*}$ & $16.1^{\mathrm{ns}}$ \\
\hline $\mathrm{T} 4$ & $-49.0^{*}$ & $93.6^{* *}$ & $110.7^{*}$ & $-43.6^{* *}$ & $79.8^{* *}$ & $18.1^{*}$ \\
\hline T5 & $-21.8^{*}$ & $75.5^{* *}$ & $9.4^{\mathrm{ns}}$ & $-22.3^{* *}$ & $2.4^{\mathrm{ns}}$ & $-9.9^{\mathrm{ns}}$ \\
\hline
\end{tabular}

Note: ${ }^{[a \mid} \mathrm{TP}=$ Tapping; Data of tapping day after stimulation (TP (+1), TP (+2) and TP (+3)) were compared with tapping day before stimulation (TP (-1)) of 5 treatments ns $=$ not signific ant, ${ }^{*}=$ signific ant $(\mathrm{P} \leq 0.05),{ }^{* *}=$ highly significant $(\mathrm{P} \leq 0.01)$

stimulation. However, PI of T4 and T5 showed significant differences on the 3 tapping days after stimulation except on the first and the third tapping days after stimulation in the high yield period. The response of AY to ethylene stimulation on the 3 tapping days after stimulation in both periods for the 5 treatments is shown in Table 8. In both periods, AY of T1 did not show a significant difference in the 2 tapping days after stimulation except on the third tapping day. But AY of T2 was significant difference on the 3 tapping days after stimulation. However, $\mathrm{T} 1$ and $\mathrm{T} 2 \mathrm{did}$ not receive any stimulation. The response to ethylene stimulation in both periods, showed that AY of T3 and T5 had similarly significant differences on the 3 tapping days after stimulation. But T4 showed the only significant difference on the 3 tapping days after stimulation in the low yield period and on the first tapping day after stimulation in the high yield period. Table 9 shows the response of Suc to ethylene stimulation on the 3 tapping days after stimulation of the 5 treatments. In both periods, Suc of $\mathrm{T} 1$ was significantly different on the 3 tapping days after stimulation. In the same manner, Suc of T2 was significantly different on the 3 tapping days after stimulation except on the third tapping day after stimulation in the high yield period. Besides, the response to ethylene stimulation showed that Suc of T3 in the low yield period showed significant difference among the taping days except on the second tapping day after stimulation. In contrast, Suc of T3 in the high yield period was not significantly different except for the second tapping day after stimulation. Suc of T4 in both periods was significantly different on the 3 tapping days after stimulation. Suc of $\mathrm{T} 5$ in the low yield period showed significant difference except on the third tapping day after stimulation, while Suc in the high yield period showed no significant difference except on the first tapping day after stimulation.

\section{Discussion}

The purpose of this study was to analyze the responses of yield and the changing parameters linked to yield potential to the ethylene stimulation. Initial flow rate (IFR) between tapping systems on each tapping day was high in the reduced tapping frequency (d3) treatments in the low yield period, but it was low in the high yield period in comparison with the traditional tapping system widely used in Thailand $S / 32 d / 3$. It seems that the differences in the periods in each season has impacted IFR. The latex flow characteristics conformed to the results of Sreelatha et al. (2007). IFR of the d 3 treatments (T3 and T5) were nearly the same though the cut length and stimulation frequency were different. Under alternate (d2) treatments, IFR of the $\mathrm{d} 2$ treatment without ethylene stimulation was higher than that of the $\mathrm{d} 2$ with stimulation treatment in both periods with a shorter cut length. IFR gradually increased during succeeding tapping days in all treatments due to the stress of tappings inducing flow and regeneration processes involving water exchanges in latex vessels (Jacob et al., 1988; d'Auzac et al., 1997). The change of IFR in both periods resulted in significant differences on each tapping day. IFR rapidly increased after ethylene stimulation because ethylene induced high turgor pressure in the latex vessel. It increased the latex flow (Jacob et al., 1988; d'Auzac et al., 1997). In the low yield period, IFR of the 5 treatments were lower than that of the high yield period (Sreelatha et al., 2007), therefore, low IFR and high PI were concomitantly shown lower in the low yield period. Plugging index (PI) among the tapping systems in both periods were high for the traditional tapping systems or non-stimulated treatments. However, they were low in the ethylene application treatments. Actually, the response of PI during succeeding tapping days decreased, resulting in the prolonged latex flow per tapping. Besides, PI suddenly decreased after ethylene stimulation in the low yield period. It gradually decreased after stimulation in the high yield period. It was clear that stimulation reduced the plugging of latex flow and resulted in increasing the latex yield after tapping (Gunasekera et al., 2013). The sudden decrease of PI resulted in high yield per tapping because of the delay of plugging of latex vessel (Jetro and Simon, 2007). In addition, ethylene 
stimulation was relatively more effective in the low yield period; starting tapping combined with high temperature and new canopy (Sreelatha et al., 2007), than the high yield period in which latex flow before stimulation was initially longer than the low yield period. Average latex yield (AY) was the highest in the $\mathrm{d} 3$ treatments with stimulation in the low yield period. While, AY of d2 and d 3 treatments were higher than the traditional tapping system in the high yielding period. In our study, the AY increased during succeeding tapping days of the 5 treatments. For the treatments with ethylene stimulation, AY rapidly increased after stimulation in both periods confirmed by Jetro and Simon (2007) regarding the response of the latex yield after stimulation on each tapping day. The half spiral cut downward with every third day tapping with stimulation 8 times per year showed superior AY per tapping in comparison with third spiral cut downward at third daily tapping with stimulation 12 times per year. This is related to the study of Traore et al. (2011) concerning the frequency of stimulation: the higher the frequency of stimulation, the lower the response at each tapping. This could impact the laticiferous system because of overstimulation. AY in the high yield period of the 5 treatments were significantly higher than in the low yield period. As observed by Sreelatha et al. (2007). Priyadarshan (2003) reported low yield during May to September and high yield during October to January in any given year. In addition, high temperature, defoliation and refoliation were also impacted on latex yield (Rao and Vijayakumar, 1992). Lower temperature $\left(23-26{ }^{\circ} \mathrm{C}\right)$ lead to high yields because they prolong the latex flow (Shuogang and Yagang, 1990). The best response to ethylene stimulation was found in the low yield period because of the relatively higher effect on the metabolism of the rubber trees at this stage. Sucrose content (Suc) before ethylene stimulation was higher in the traditional tapping systems than in other tapping systems. But after stimulation on the first tapping day, Suc of half spiral cut downward with $\mathrm{d} 3$ frequency only increased and was the highest in both periods as a result of ethylene on the processes of sucrose transportation in the latex cells (Jacob et al., 1989; d'Auzac et al., 1997; Lacote et al., 2010; DusotoitCoucaud et al., 2009). The third spiral cut downward (S/3) treatments recovered more Suc than the other treatments because ethylene application inducing sucrose loading in latex cells and utilization resulted in increased rubber biosynthesis and yield. However, Suc content between both periods showed that Suc in the low yield period was higher than in the high yield period. Sreelatha et al. (2007) reported that an activator of sucrose synthase was high in the low yielding season, and resulted in an increase of sucrose available for both physiological processes in the trees: completed foliage, and yield due to the restarting of tapping. In high yield period the lower Suc metabolism dedicated more to rubber biosynthesis creating a sink for sucrose content has to be connected with the corresponding (Jetro and Simon, 2007). The result was could show the effectiveness of stimulation in the low yield period.

\section{Materials and Methods}

\section{Experimental site and plant material}

These experiment were carried out at the Thepa Research Station (6 $68^{\prime} 0.7^{\prime \prime} \mathrm{N} 100^{\circ} 56^{\prime} 37.2^{\prime \prime} \mathrm{E}$, altitude $33 \mathrm{~m}$ above sea level) Thepa district, Songkhla province in southern Thailand. The climate is tropical with two seasons, a dry season and a rainy season (Sternstein, 1962; TMD, 2013). The dry season is from mid-February to mid-May, and the rainy season is from mid-May to mid-February. RRIM 600 clones were used in the experiment. Rubber trees were planted ( $7 \times 3 \mathrm{~m}$ spacing) in 2005 , and they were selected before tapping by homogenous girth at $1.70 \mathrm{~m}$ from the ground. The tapping was opened at $1.50 \mathrm{~m}$ from the ground on panel BO-1. The experiments were started in August 2013 and the study was focused on the period between May and October 2014.

\section{Experimental treatments and design}

The experiment was arranged as One Tree Plot design (Sdoodee et al., 2012) with five treatments comprising three trees per treatment. Protocol of the study treatments is presented in Table 1 according to Vijayakumar et al. (2009) international notation.

The response of the trees to the ethylene stimulation (ethephon) was studied in each tapping day before and after stimulation. The tapping agenda is presented in Table 2 . The experiments were made in two periods, low yield period (LY) and high yield period (HY). LY was in May when tapping was started. HY was in October, in the rainy season, when the yield at each tapping is usually high.

\section{Data collection and analysis}

Latex yield (gram per tree per tapping: g/t/t) was calculated from each tree by weighting the latex yield at each tapping. Total solid content was measured from a bulk sample taken in each treatment in order to convert fresh weights in grams of dry rubber per tree and per tapping. Initial flow rate and plugging index were calculated according to the methods of Milford et al. (1969). The initial flow rate was collected in the first 5 minutes, expressed in milliliters per minute $(\mathrm{ml} / \mathrm{min})$ and calculated following the formula:

Initial flow rate $=\frac{\text { volume of latex first five minutes }}{5}$

Plugging index was calculated following the formula:

$$
\text { Plugging index }=\left(\frac{\text { Initial flow rate }}{\text { Volume of latex }}\right) \times 100
$$

The main latex biochemical parameters, i.e. sucrose (Suc) content, was measured in a latex sample of 1 milliliter in the first 5 minutes of the flow after tapping, from each tree taken in each treatment, using methods developed by CIRAD (Jacob et al., 1989) adapted in 1995 by IRRDB (1995). Sucrose content was expressed in millimoles of latex (mM) and updated for Thailand by Gohet and Chantuma (1999). The data was statistical analyzed by DSAASTAT v 1.1 package software (Onofri, 2007).

\section{Conclusion}

Ethylene application has affected all the parameters linked to potential yield of the trees. The initial flow rate (IFR), plugging index (PI), average latex yield (AY) and sucrose content (Suc) of rubber clone RRIM 600 under low frequency tapping systems were significantly affected. There was significant difference among the tapping systems during the low yield and the high yield periods. It showed that the response to stimulation was dependant on the physiological status of the trees according to the seasonal variation of the tree metabolism in the low and high yield periods. The yield was always higher in the high yield period than in the low yield period, even the relative higher response to stimulation on IFR, PI and Suc were seen in the low yield period. But a more sustainable positive effect of stimulation is expected in the yield period corresponding to metabolism dedicated more 
to rubber biosynthesis creating a sink for sucrose. In any case, the treatments in $\mathrm{d} 3$ tapping frequency with stimulation showed drastic changes in sucrose content. It is remarkable, that in Thailand, even in a heavy rainy area, it is possible to use ethylene stimulation to increase the potential yield of the trees at each tapping.

\section{Acknowledgments}

This work was supported by Prince of Songkla University and Graduate Scholarship. This research was conducted under the Hevea Research Platform in Partnership (HRPP), Thailand. We are appreciated of CIRAD for research guidance and technology support.

\section{References}

Buttery BR, Boatman SG (1964) Turgor pressures in phloem: measurements on Hevea latex. Science. 145:285-286.

Buttery BR, Boatman SG (1966) Manometric measurement of turgor pressures in laticiferous phloem tissues. J Exp Bot. 17:283-296.

d'Auzac J, Jacob JL, Prévôt JC, Clément A, Gallois R, Chrestin H, Lacote R, Pujade-Renaud V Gohet E (1997) The regulation of cis-polyisoprene production (natural rubber) from Hevea brasiliensis. In: Pandalai SG (ed) Recent Research Developments in Plant Physiology, vol 1. Trivandrum, pp. 273-332.

Dusotoit-Coucaud A, Brunel N, Kongsawadworakul P, Viboonjun U, Lacointe A, Julien JL, Chrestin H, Sakr S (2009) Sucrose importation into laticifers of Hevea brasiliensis, in relation to ethylene stimulation of latex production. Ann Bot. 104:635-647.

Gohet E, Chantuma P (1999) Micro Latex Diagnosis. (Standard Analysis Procedure) Microdiagnostic Latex Training. Chachoengsao Rubber Research Center, RRITDOA-MOAC. 22-26 November 1999, p 80.

Gunasekera HKLK, De Costa WAJM, Nugawela A (2013) Effect of opening girth and some latex physiological parameters on yield of Rubber (Hevea brasiliensis). Int J Innov Appl Studies. 4:88-100.

Gunasekera HKLK, Nugawela A, De Costa WAJM, Attanayaka DPSTG (2002) Exploitation systems for some Hevea brasiliensis Muell. Arg. Clones for improved economic performance. Trop Agric Res. 14:128-137.

IRRDB (1995) Manual of biochemical and physiological tests. Ref. 1995/3, January 26.

Jacob JL, Prévôt JC, Roussel D, Lacrotte R, Serres E, d'Auzac J, Eschbach JM, Omont H (1989) Yield-limiting factors, latex physiological parameters, latex diagnosis and clonal typology. In: d'Auzac J, Jacob JL, Chrestin H (eds) Physiology of Rubber Tree Latex, Boca Raton, CRC Press, pp. 345-403.

Jacob JL, Serres E, Prevot JC, Lacrotte R, Clement-Vidal A, Eschbach JM, Omont H (1988) Mise au Point du Diagnostic Latex. Agritrop. 12:97-118.

Jetro NN, Simon GM (2007) Effect of 2chloroethylphosphonic acid formulations as yield stimulants on Hevea brasiliensis. Afr J Biotechnol. 6:523528.

Kudaligama KVVS, Rodrigo VHI, Fernando KMEP, Yapa PAJ (2010) Response of low frequency harvesting systems of rubber under drier climatic conditions in Sri LanKa. In: Proceedings of the 15th International Forestry and Environment Symposium, University of Sri Jayewardenepura, Sri Lanka, 26-27 November 2010, pp. 62-69.
Lacote R, Doumbia A, Obouayeba S, Gohet E (2013) Sustainable rubber production through good latex harvesting practices: stimulation based on clonal latex functional typology and tapping panel management. In: IRRDB Workshop on Latex Harvesting Technology, RRIV Binh Duong, Vietnam November 19th - 22nd.

Lacote R, Gabla O, Obouayeba S, Eschbach JM, Rivano F, Dian K, Gohet E (2010) Long-term effect of ethylene stimulation on the yield of rubber trees is linked to latex cell biochemistry. Field Crop Res. 115:94-98.

Mesquita AC, Oliceira LEM, Mazzafera P, Delú-Filho N (2006) Anatomical characteristics and enzymes of the sucrose metabolism and their relationship with latex yield in the rubber tree (Hevea brasiliensis Muell. Arg.). Braz J Plant Physiol. 18:263-268.

Milford GFJ, Paardekooper EC, Ho CY (1969) Latex vessel plugging, its importance to yield and clonal behaviour. J Rubb Res Inst Malaysia. 21:274-282.

Nakkanong K, Nualsri C, Sdoodee S (2008) Analysis of genetic diversity in early introduced clones of rubber tree (Hevea brasiliensis) using RAPD and microsatellite markers. Songklanakarin J Sci Technol. 30:553-560.

Njukeng JN, Muenyi PM, Ngane BK, Ehabe EE (2011) Ethephon stimulation and yield response of some Hevea clones in the humid forests of south west Cameroon. Int $\mathbf{J}$ Agron. 2011:1-5

Onofri A (2007) Routine statistical analyses of field experiments by using an Excel extension. In: Proceedings 6th National Conference Italian Biometric Society: La statistica nelle scienze della vita e dell'ambiente, Pisa, Italy, 20-22 June 2007, pp. 93-96.

Prasanna WRAC, Rodrigo VHI, Abeysinghe DC, Kudaligama KVVS (2010) Stimulant levels to be used with two low intensity harvesting $(\mathrm{LIH})$ systems of rubber under wet and intermediate zones of Sri Lanka. In: Proceedings of the 15th International Forestry and Environment Symposium. University of Sri Jayewardenepura, Sri Lanka, pp. 265-272.

Pethin D, Nakkanong K, Nualsri C (2015) Performance and genetic assessment of rubber tree clones in Southern Thailand. Sci Agric. 72:306-313.

Priyadarshan PM (2003) Contributions of weather variables for specific adaptation of rubber tree (Hevea brasiliensis Muell- Arg) clones. Genet Mol Biol. 26:435-440.

Rao PS, Vijayakumar KR (1992) Climatic Requirements. In: Sethuraj MR, Mathew NM (eds) Natural rubber: Biology, Cultivation and Technology, Amsterdam: Elsievier, Science Publishers.

Sdoodee S, Leconte A, Rongsawat S, Rukkhun J, Huaynui T, Chinatiam H (2012) First tests of "Double Cut Alternative" rubber tapping system in Southern Thailand. Kasetsart J (Nat Sci). 46:33-38.

Shuogang A, Yagang G (1990) Extrapolation of the high yield physiological regulation of Hevea brasiliensis in Xishuangbanna. In: Proceedings of IRRDB symposium on Physiology and Exploitation of Hevea brasiliensis, pp 8392. Kunming, China, 1990.

Soumahin EF, Obouateba S, Anno PA (2009) Low tapping frequency with hormonal stimulation on Hevea brasiliensis clone PB 217 reduces tapping manpower requirement. J Anim Plant Sci. 2:109-117.

Soumahin EF, Obouateba S, Dick KE, Dogbo DO, Anno AP (2010) Low intensity tapping systems applied to clone PR 107 of Hevea brasiliensis (Muell. Arg.): Results of 21 years of exploitation in south-eastern Cote d'Ivoire. Afr J Plant Sci. 4:145-153. 
Sreelatha S, Simon SP, Kurup GM, Vijayakumar KR (2007) Biochemical mechanisms associated with low yield during stress in Hevea clone RRII 105. J Rubber Res. 10:107-115.

Sternstein L (1962) The Rainfall of Thailand "A Study by Lawrence Sternstein" supported by The U.S. Army Quartermaster Corps. In: Research and Engineering Command, Project No.7-83-01-006.

TMD (2013) World's season. Thai Meteorological Department, Thailand.

Traore MS, Diarrassouba M, Okoma KM, Dick KE, Soumahin EF, Coulibaly LF, Obouayeba S (2011) Longterm effect of different annual frequencies of ethylene stimulation on rubber productivity of clone GT1 of Hevea brasiliensis (Muell. Arg.) in south east of Cote d'Ivoire. Agric Biol J N Am. 2:1251-1260.

Tupy J (1973) The level and distribution pattern of latex sucrose along the trunk of Hevea brasiliensis Mull.Arg. as affected by the sink region induced by latex tapping. Physiol Veg. 11:1-11.
Tupy J (1985) Some aspects of sucrose transport and utilization in latex producing bark of Hevea brasiliensis. Planta. 27:51-64.

Vijayakumar KR, Gohet E, Thomas KU, Xiaodi W, Sumarmadji, Rodrigo L, Thanh DK, Sopchoke P, Karunaichamy KSTK, Said MA (2009) Special communication: Revised international notation for latex harvest technology. J Rubber Res. 12:103-115.

Waidyanatha UP, Pathiratne LSS (1971) Studies on latex flow patterns and plugging indices of clones. J Rubb Res Inst Sri Lanka. 48:47-55.

Yeang HY (2005) The kinetics of latex flow from the rubber tree in relation to latex vessel plugging and turgor pressure. J Rubber Res., 8:160-181. 